\title{
Carnet de route de Jordanie. De la recherche de terrain à l'expérience de la médiation Partie 2
}

\section{Géraldine Chatelard}

\section{(2) OpenEdition \\ 12 Journals}

\section{Édition électronique}

URL : http://journals.openedition.org/conflits/854

DOI : $10.4000 /$ conflits.854

ISSN : $1777-5345$

Éditeur :

CCLS - Centre d'études sur les conflits lilberté et sécurité, L'Harmattan

Édition imprimée

Date de publication : 1 septembre 2002

ISBN : 2-7475-3432-4

ISSN : 1157-996X

\section{Référence électronique}

Géraldine Chatelard, «Carnet de route de Jordanie. De la recherche de terrain à l'expérience de la médiation Partie 2 », Cultures \& Conflits [En ligne], 47 | automne 2002, mis en ligne le 29 avril 2003, consulté le 30 mars 2021. URL : http://journals.openedition.org/conflits/854 ; DOI : https://doi.org/ $10.4000 /$ conflits.854

Ce document a été généré automatiquement le 30 mars 2021.

Creative Commons License 


\title{
Carnet de route de Jordanie. De la recherche de terrain à l'expérience de la médiation Partie 2
}

\author{
Géraldine Chatelard
}

1 Deux jours plus tard, aller-retour à Amman avec Tony, Di et Abou Yasser. Nous allons rendre visite au Prince Mir'ed, jeune cousin du roi qui a soutenu les bédouins il y a trois ans pour empêcher que leur village ne soit déplacé. Nous avons été recommandés par Raouf Dabbas, le président d'une autre ONG qui s'occupe d'environnement, qui connaît bien Son Altesse et qui nous accompagne. Il a d'ailleurs lui-même failli épouser une princesse. Toujours aussi civile, la société jordanienne... Mais Raouf à le mérite de se déplacer régulièrement à Ramm, n'hésite pas à tremper les doigts dans le plat de mensaf, et joue fort bien son rôle dans le système de wasta, version locale du clientélisme généralisé, qui, à défaut de réelle société civile, permet de faire remonter au sommet de l'Etat les revendications de la base. L'altesse a une oreille attentive. Deux jours plus tard, encore, après avoir bu force thé sous les tentes et dans le village pour relater au plus grand nombre les discussions avec le Douktour et l'altesse, été contrainte à mon corps défendant de prouver mon mépris pour la RSCN en suivant une chasse à la gazelle, et surtout promis aux bédouins qu'on allait continuer la lutte ensemble, je suis rentrée en Italie.

Mai 2002

2 A nouveau loin du terrain, il est peut-être temps de me demander pourquoi j'en suis arrivée à m'impliquer dans la médiation entre les bédouins et avec les acteurs institutionnels. Le «comment» me semble moins problématique, résultant de l'enchaînement de propositions et de sollicitations liées à mon statut de chercheuse. A toutes les étapes, j'ai eu le choix de répondre par la négative, jugeant que mon engagement outrepassait ce rôle. J'ai pourtant accepté d'utiliser mes connaissances et mes compétences non plus pour produire du savoir, mais à des fins pratiques d'intervention dans le monde de mes enquêtés. 
3 Se pose tout d'abord la question de ma relation à ce que j'ai construit comme un objet de recherche, de mon implication préalable dans un espace social circonscrit comme un terrain par mon enquête. Je ne suis pas arrivée à Wadi Ramm comme anthropologue, je n'ai jamais vraiment fait "d'entrée sur le terrain ». J'ai commencé à fréquenter Ramm comme touriste il y a presque dix ans, y suis revenue souvent comme visiteuse résidente étrangère à Amman, ai fini par y accompagner des touristes, jouant un rôle social et économique dans un site que j'ai ensuite décidé d'étudier en abandonnant mes autres casquettes. Mon engagement était celui d'une actrice avant d'être celui de chercheuse. La décision de mise à distance a été mienne, elle n'a pas impliqué, de la part des autres acteurs, une démarche similaire. Il n'a d'ailleurs pas été facile pour tous les habitants de Ramm de s'habituer à ma nouvelle identité. En la matière, le titre et le statut de «docteur» m'ont permis de m'extraire des enjeux locaux liés au tourisme. Mais ils n'ont pas dénoué les liens d'amitié qui me liaient aux personnes, pas plus qu'entamé la relation symbolique d'adoption au sein d'une famille élargie.

Ma position reste problématique à Ramm. Comme les touristes, l'anthropologue est là pour observer, mais il cherche à produire un savoir construit de façon systématique et fondé sur des preuves empiriques plutôt qu'à avoir des expériences hédonistes. Il a la prétention de pénétrer des lieux dont les touristes sont exclus et d'accéder au statut d'« étranger de l'intérieur », comme il en existe certains, sans pour autant s'impliquer dans les dynamiques économiques ou sociales locales. De fait, ce qui pose problème, c'est l'asymétrie entre ce que je sollicite des mes interlocuteurs et ce que je leur restitue. Bien sûr, je donne à Ramm des cadeaux en tous genres, du temps à discuter de l'Europe ou à apprendre aux femmes un peu d'anglais, en somme de simples marques d'amitié. Je fais aussi parfois à mes interlocuteurs un résumé oral de mes conclusions de recherche, ce qui relance les discussions, mais ces comptes-rendus sont toujours amputés de la complexité des analyses plus abstraites. Peu sont ceux qui comprennent les objectifs de ma recherche et on m'interroge souvent sur le pourquoi. Une première réponse à la question de mon engagement est peut-être à trouver dans le fort sentiment que j'ai de l'inégalité dans l'échange. A défaut de pouvoir restituer aux habitants de Ramm ma vision complète et analytique de leur réalité sociale ou de les convaincre que je suis motivée par un simple intérêt scientifique, je me suis sentie tenue de leur offrir mes compétences lorsqu'ils ont estimé en avoir besoin. D'assumer totalement le rôle social que l'on m'attribue localement de détentrice d'un savoir particulier et d'interface entre plusieurs groupes dont je maîtrise les codes. Ce rôle n'est, à vrai dire, pas si éloigné de la pratique de la traduction anthropologique, bien que celle-ci soit à sens unique. Si je m'y refusais, les gens de Ramm m'auraient peutêtre retiré leur respect et leur amitié. Pour une part, mon engagement est sans doute une stratégie de préservation de l'accès au terrain.

5 Il ne se résume pourtant pas à cela. Plus profondément, il relève d'un sens de l'obligation morale. Cette situation de conflit m'apparaît critiquable d'un point de vue éthique parce que préjudiciable aux bédouins, dans une situation de vulnérabilité par rapport à des enjeux qui leur échappent. A Ramm, le tourisme international et les idéologies du développement ou de la protection de l'environnement exercent une violence symbolique et une domination économique sur les autochtones. Ils reproduisent des attitudes coloniales et un discours qui les légitime. Les bédouins ont du mal à saisir la complexité et l'étendue des forces qui les contraignent. Ils n'ont pas non plus conscience de l'existence d'outils légaux ou de réseaux internationaux qui 
puissent donner force à leurs revendications. Mon rôle alors peut-il se limiter cyniquement à assister à leur défaite annoncée et à la décrire, alors que je sais où localiser les ressources nécessaires à leur lutte pour la survie? Une responsabilité ne m'incombe-t-elle pas précisément du fait de mes connaissances universitaires? Mes convictions morales ne me dictent-elles pas d'assister un collectif en danger et d'offrir mes services pour faciliter sa résistance? Dans une certaine mesure, je ne fais que renverser ma fonction de médiation. L'anthropologie est avant tout un travail de traduction de l'expérience de l'altérité. Aux bédouins qui m'ont patiemment enseigné leur monde et initié à cette altérité, je me dois en retour de rendre intelligible d'autres visions du monde, d'analyser les stratégies de sujétion dont il sont les victimes et de leur donner les moyens de se battre à armes égales avec leurs adversaires.

6 C'est le contexte historique et idéologique colonial qui a nourri l'émergence de l'anthropologie. Cet effort de compréhension des sociétés dites "primitives» ou «traditionnelles » a été mis à profit par les Etats et les capitalistes occidentaux, avec la complicité plus ou moins directe des scientifiques, pour contrôler ces sociétés sous couvert de modernisation. Dégagée du contexte colonial et enrichie d'une réflexion épistémologique sur ses genèses et ses usages, l'anthropologie, comme les autres sciences sociales, n'en continue pas moins à produire des résultats dont l'utilisation échappe à l'anthropologue. Pour une part, comme en témoignent les volets socioéconomiques des projets de la Banque mondiale qui citent abondamment les travaux universitaires, ces résultats entretiennent encore des situations de domination et de spoliation. Mais l'anthropologue peut également s'engager du côté des acteurs qui subissent cette domination, augmenter leurs capacités de compréhension et d'action, restaurer la balance du pouvoir qui leur est défavorable. Les collègues rencontrés lors de la conférence de Dana qui m'ont conseillé sur la situation à Ramm se retrouvent également dans ce travail de défense des droits et de renforcement de la puissance (empowerment) des plus démunis. Un exemple récent est fourni par le cas des grands projets hydrauliques en Turquie du sud-ouest. Des anthropologues britanniques ont accepté de contribuer au montage d'un dossier qui a permis à une association de défense des droits de l'homme d'intenter un procès au Parlement britannique. Celui-ci avait voté l'octroi de fonds pour la construction d'un barrage qui allait causer le déplacement forcé de plusieurs milliers de villageois kurdes. La Grande-Bretagne a été contrainte de retirer son financement. Le projet a été gelé. Le problème des chercheurs qui répondent à ces sollicitations est qu'ils deviennent généralement indésirables aux yeux des autorités du pays où se situe leur terrain. C'est un risque qui ne m'échappe pas. Un autre enjeu majeur de mon engagement aux côtés des habitants de Ramm est celui de la maîtrise des résultats de mon action. Il est possible que la dynamique que j'enclenche leur soit finalement plus défavorable que bénéfique, en particulier s'ils choisissent d'aller en justice. La règle du jeu est alors de les tenir parfaitement au courant de ce que j'anticipe comme scénarios possibles et d'être prête à assumer les conséquences de mes actions. Dans tous les cas, c'est toujours de responsabilité qu'il s'agit.

7 Qu'en est-il alors du statut de l'enquête anthropologique, lorsque celle-ci rend compte d'un processus dans lequel le chercheur est activement impliqué? La question de l'objectivité de l'anthropologue est déplacée lorsqu'il joue un rôle actif d'agent du changement social. Le chercheur en sciences sociales ne se départit jamais de ses croyances personnelles, de ses connivences politiques ou de ses convictions morales, et son regard est orienté par les problématiques qu'il endosse, les questions qu'il se pose 
et les hypothèses qu'il se donne. La seule chose qu'il puisse faire est de rendre ces croyances, connivences et convictions et ces problématiques, questions et hypothèses aussi transparentes que possible. De surcroît, l'engagement sur le terrain est avant tout d'ordre pratique : il est fait de prises de contact, de gestion d'interactions, de définition de situations, de résolution de problèmes matériels, de coopération entre enquêteur et enquêtés et de conduite d'opérations d'investigation. Il résulte de décisions qui ont une portée morale et politique, il requiert des jugements sur le bien et sur le juste. A des degrés divers, le chercheur, par sa présence même, perturbe toujours le site d'enquête qu'il observe et décrit et fait surgir des questions qui n'auraient pas eu de raison d'être en son absence. Dans la tradition ethnologique française, il est pourtant rare que cette forme d'implication fasse l'objet d'une réflexion intégrée à l'enquête. Cette réflexivité est pourtant nécessaire. Le savoir en sciences sociales n'existe pas en dehors des pratiques de production de ce savoir et reste irréductiblement porteur d'une dimension éthique et politique. In fine, la reconnaissance de ces éléments en fait un savoir moins désincarné, plus ouvert à la critique.

\section{INDEX}

Index géographique : Moyen-Orient

Mots-clés : sociologie 